\title{
QOL IMPROVES AFTER
}

ADRENALECTOMY

The quality of life (QOL) of patients with unilateral primary aldosteronism improves after unilateral laparoscopic adrenalectomy, according to an Australian research group.

The clinical benefits of unilateral adrenalectomy for unilateral primary aldosteronism include cure or improvement in hypertension, but very little work has assessed the pyschosocial effects of the surgery.

Lead researcher Michael Stowasser of the University of Queensland Medical School explains what prompted the research. "Patients with hypertension associated with unilateral primary aldosteronism appeared to exhibit more irritability and emotionality than patients with hypertension from other causes, then after surgery, patients and family members reported comparative calmness."

The researchers enrolled 22 patients (14 men) undergoing laparoscopic unilateral adrenalectomy for unilateral primary aldosteronism in the 1-year pilot study. Blood pressure, biochemical parameters and QOL were measured before and after surgery. QOL was selfassessed using the SF-36 questionnaire.

Surgery either cured or improved hypertension, normalized hypokalemia and reduced medication needs. In the presurgery assessment, QOL was reduced for patients compared with the general population. At 3 months postsurgery, SF-36 scores in all eight domains had risen to a normal level, and this level was maintained at 6 months.

Although the researchers concede that patient anxiety before surgery might have partially influenced the results, the findings, nevertheless, suggest that "patients generally feel better postoperatively, and for the patient and their family, this is often the most important outcome of all," concludes Stowasser.

Claire Greenhill

Original article Sukor, N. et al. Improved quality of life, blood pressure, and biochemical status following laparoscopic adrenalectomy for unilateral primary aldosteronism. J. Clin. Endocrinol. Metab. 95, 1360-1364 (2010) 